\title{
Assessing the impact of a construction virtual reality game on design review skills of construction student
}

Article

Accepted Version

Kandi, V. R., Castronovo, F., Brittle, P., Mastrolembo Ventura, S. and Nikolic, D. (2020) Assessing the impact of a construction virtual reality game on design review skills of construction student. Journal of Architectural Engineering, 26 (4). ISSN 1943-5568 doi: https://doi.org/10.1061/ (ASCE)AE.1943-5568.0000434 Available at https://centaur.reading.ac.uk/93660/

It is advisable to refer to the publisher's version if you intend to cite from the work. See Guidance on citing.

Published version at: https://ascelibrary.org/doi/10.1061/\%28ASCE\%29AE.1943-5568.0000434 To link to this article DOI: http://dx.doi.org/10.1061/(ASCE)AE.19435568.0000434

Publisher: ASCE

All outputs in CentAUR are protected by Intellectual Property Rights law, including copyright law. Copyright and IPR is retained by the creators or other copyright holders. Terms and conditions for use of this material are defined in the End User Agreement. 


\section{CentAUR}

Central Archive at the University of Reading

Reading's research outputs online 


\title{
ASSESSING THE IMPACT OF A CONSTRUCTION VIRTUAL REALITY GAME ON DESIGN REVIEW SKILLS OF CONSTRUCTION STUDENTS
}

\author{
Varsha Reddy Kandi ${ }^{1}$; Fadi Castronovo ${ }^{2}$; Patrick Brittle ${ }^{3}$; Silvia Mastrolembo Ventura ${ }^{4}$; Dragana \\ Nikolic ${ }^{5}$
}

1 Graduate Student, Construction Management Program, School of Engineering, California State University, East Bay, Hayward, CA 94542; Email vkandi2@horizon.csueastbay.edu

2 Assistant Professor, Construction Management Program, School of Engineering, California State University, East Bay, Hayward, CA 94542; Email fadi.castronovo@csueastbay.edu

3 Assistant Professor, Construction Management Program, College of Engineering, Computer Science \& Construction Management, California State University, Chico, Chico, CA 95929; Email pbrittle@csuchico.edu

4 Research Fellow, Department of Civil, Environmental, Architectural Engineering and Mathematics, University of Brescia, Brescia, Italy; Email silvia.mastrolemboventura@unibs.it

5 Lecturer in Digital Architecture, School of Construction Management and Engineering, University of Reading, Reading, UK; Email d.nikolic@,reading.ac.uk

\begin{abstract}
Being able to perform an effective design review is a fundamental skill that every design or construction student should possess. Performing a design review requires critical thinking, analysis, and communication skills. To support the acquisition of these skills, this study investigated the impact of a virtual reality game, the design review simulator (DRS), in a classroom environment. To assess the impact of the game, the authors performed a crossover experiment with 120 students. The students were split into groups and were asked to perform design reviews in two treatment conditions, 2D drawings or the DRS. A knowledge test was administered at the end of different treatments to collect quantitative data on the students' performance. Results from this research indicate that the DRS supported students in identifying a higher number of design mistakes. Furthermore, students that played with the DRS after performing 2D drawings reviews scored significantly higher in the knowledge test. The research results contribute to the growing knowledge base on the implementation of VR in the classroom and in supporting students in meeting learning objectives related to design review skills. In particular, the research illustrated that the benefits of VR found in the construction industry in terms of improved communication, user involvement, and feedback collection could be translated into the classroom environment.
\end{abstract}

\section{INTRODUCTION}

An effective design review process is critical to the successful delivery of a construction project. Many of the cost, schedule and quality-related problems encountered during construction 
projects are the direct result of errors or inadequacies in the design and construction deliverables. The design review process has to minimize these issues. When design reviews are performed early and regularly in the design stages of the project, this effort can play an important role in limiting cost and schedule overruns (Kirby et al. 1989), as well as in guaranteeing functionality and effectiveness for the proposed solution. Simply put, the design review process consists of reviewing construction documents for accuracy, completeness, and correctness with the intent of finding omissions, ambiguities, and inadequacies in the design (Kirby et al. 1989).

In this context, equipping construction management students with such design review skills becomes critical. The American Council for Construction Education (ACCE), which provides accreditation to construction management programs, reinforces the importance of students being proficient in performing design reviews. According to ACCE's criteria, "the Constructor is to have an understanding of the contribution of the design discipline's processes" and "must be able to communicate with the design professionals, and should be capable of participating during the planning phase of design-build projects" (ACCE 2014). Yet, teaching such design review skills presents educators with challenges.

Traditionally, design reviews are conducted by reviewing the project design through reading $2 \mathrm{D}$ drawings. Reading design and construction plans requires the ability to interpret all the necessary information by referencing various drawing views, such as plan views, sections, and details among others. This effort relies on the professional training and the astuteness of the reviewers, drawing on their past working experiences (Khemlani et al. 1998). The inherent demands of reading project drawings are especially challenging for the entry-level undergraduate construction management students who often do not yet have any professional construction experience and still may lack skills to fully understand technical representations.

Previous research showed how advanced and interactive visualization technologies, such as virtual reality (VR), could support students and industry professionals in performing design reviews (Whyte 2003). In fact, VR has shown to provide value for project teams through encouraging effective communication, and improving both the productivity of design review meetings, as well as user involvement and the feedback collection from these meetings, (van den Berg et al. 2017; Lather 2014).

In order to understand how VR could support construction students in performing design reviews if compared with more traditional representations, the authors adopted a quantitative and comparative research approach, recruiting a large sample size of undergraduate students. The students participated in an experiment where they were compared for their ability to identify design mistakes using immersive VR technology and a paper-based method. The results of this study provide valuable information for educators on the value of VR in teaching construction management students design review skills.

\section{LITERATURE REVIEW}

\subsection{VIRTUAL REALITY IN DESIGN REVIEWS}

"Design review is one of the most important processes in architectural and engineering design" (van den Berg et al. 2017). A design review is a milestone, within the design process, whereby a proposed solution is evaluated in order to verify the outcome of the design intent, identify possible failures with respect to program, and evaluate previously defined needs and project requirements, such as the function of spaces or their overall performance (Castronovo et 
al. 2013; Liu et al. 2020). Finally, based on the results of such an evaluation, stakeholders decide upon any necessary changes. Design reviews are crucial for 1) timely detecting and identifying discrepancies, errors and inconsistencies in design (i.e., the anticipation paradigm) (Shiratuddin and Thabet 2007) and 2) allowing stakeholders to discuss and take decisions as a group (i.e., the collaboration paradigm) (Le Dantec and Do 2009).

The specific objectives of design review, as well as the stakeholders involved, vary depending on the design stage. As the design evolves, the goals of the design review tend to validate and refine the design concepts (Liu 2017; Liu et al. 2018). However, the process of either exchange or transfer of information is complicated, and visualization techniques (e.g., sketches, drawings, mock-ups, renders) play an important role in the complex interaction between stakeholders with different backgrounds and technical expertise. Design review meetings typically use traditional forms of representations, such as $2 \mathrm{D}$ drawings that are limited in "information transferability" (van den Berg et al. 2017). For example, it is difficult to imagine how a design intent will look like when realized by just looking at traditional representations (Castronovo et al. 2013). Moreover, people without technical expertise usually find it difficult to perceive the spaces on 2D drawings, renderings, or video-renderings, thus causing an incomplete understanding of proposed design solutions (van den Berg et al. 2017).

With the advancement of building information modelling (BIM) procedures and technologies that center on 3D model development, research into the design review process has shifted focus to the use of advanced visualization technologies, such as virtual reality models, or immersive and interactive displays (Liu et al. 2018). A building information model could be the starting point for the development of a virtual facility prototype, which implies the adoption of virtual reality systems as visualization and interaction platform (Mastrolembo Ventura et al. 2019). Most VR environments are primarily based on visual interaction, using a range of display configurations such as computer screens or stereoscopic projection displays as output systems (Whyte and Nikolic 2018). Additional sensory information could be eventually provided through haptic devices, speakers, headphones, and olfactic technologies (Li et al. 2018, Bordegoni and Rizzi 2011).

VR systems are considered to be enablers for collaboration in design review and stakeholder engagement (Castronovo et al. 2013; Lather and Messner 2018; Leicht 2009). These systems provide multiple stakeholders, whether they are collocated or remote, with a dynamic and near-full scale 3D visualization in a shared problem-solving environment (Boton 2018). As a result, this process increases their level of engagement in the decision-making process (Bullinger et al. 2010), providing an opportunity for all the project stakeholders to collaborate for an extended review when design issues are detected and resolved systematically (Schaumann et al. 2016). Liu et al. (2014) studied the impact of using projection-based immersive virtual reality to support integrated design review processes and showed that VR models allowed reviewers to understand more quickly or easily the implication of actual design decisions. Other research also highlighted that an interactive environment such as VR can significantly enhance team communication and collaboration during design review in (Bassanino et al. 2014; Dunston et al. 2011; Majumdar et al. 2006). VR systems also allow anticipating design decisions and identifying design issues that would not be tracked otherwise (Okeil 2010). Furthermore, VR systems could promote high levels of involvement and optimize the learning process (Faas et al. 2014) by representing building proposal in a more intuitive "experiential-spatial" manner resembling the real world and thus, more closely matching the demand of end-users (Paes et al. 2017). 
Empirical studies offer evidence how first-person experience and sense of presence -- key attributes of VR experience -- support design reviews. Moreover, previous studies discussed how immersion, interaction, and user involvement also offer potential in education by making learning more engaging (Freina and Ott 2015; Jensen and Konradsen 2018). Education-related VR use cases represent one of the main purposes for VR adoption in design and construction (Lather and Messner 2018). For example, VR has been explored for its effects on engagement, enjoyment, or memory (Lin et al. 2002) or as a means of interactive storytelling (Cavazza et al. 2004) as a way to support dynamic and more active learning. At the same time, VR has an inherently three-dimensional and interactive environment that can support the students in design and engineering disciplines who are required to build their spatial reasoning skills (Castronovo et al. 2019).

In the past, the use of immersive VR in education has been limited due to high prices of the devices and their limited usability. Currently, commercial VR devices such as "Oculus Rift" make it easier to access and apply VR in many educational situations (Freina and Ott 2015). At the same time, the need to assess the impact of VR compared to traditional visualization techniques still exists. In particular, the number of VR-related research is "drastically lower in the construction domain than in other industries", such as product design and computer science (Paes et al. 2017). Moreover, Sacks et al. (2013) stated that the successful adoption of VR technologies in other industries cannot prove its effectiveness for architectural and construction practices, which has not been rigorously tested yet. According to a rising research trend, a more comparative and quantitative approach is needed in VR-related studies in order to understand and quantify the impact of virtual reality on user performance (Khashe et al. 2018; Paes et al. 2017). Performing this research is a necessary step to its wider adoption in the construction industry and so, to justify further investments (Khashe et al. 2018; Paes et al. 2017).

\subsection{EDUCATIONAL GAMES IN CONSTRUCTION}

In the context of design and construction education, improving students' analytical and problem-solving skills has been the main driver for exploring innovative and interactive learning experiences. At the core of these skills, on the one hand, is the ability to visualize the design information in order to design and evaluate proposed solutions, which is typically challenging for undergraduate students. Visualization skills are seen as part of what is broadly defined as spatial cognition, which in the design context has been variously described as one's understanding of the proportions of a given space (Pinet 1997), wayfinding or one's ability to orient in a given space, or the relationship between various spaces (Henry and Furness 1993). The implication for fostering the development of such skills in students is to complement the use of static, abstract, and two-dimensional representations, deemed to be limiting, with those that are more intuitive, interactive and multi-dimensional. On the other hand, broader issues challenging the current methods of teaching design and construction students center around the idea of achieving professional expertise, or the ability to apply relevant domain knowledge in novel situations (Litzinger et al. 2011). This all-encompassing concept of professional expertise further invites considerations of student motivation, engagement, and deeper learning. As a result, more handson and problem-based learning approaches aligned with real-life scenarios are seen to offer a more effective learning experience that can also maximize the impact of time students spend working on them (Williams and Pender 2002). 
Problem-based or project-based learning approaches, informed by broader theories of situated cognition and active learning, view knowledge as an interaction within a specific context, placing emphasis not on memory, but perception. As a result, visual and interactive computer-based learning environments have been steadily viewed as a promising way for students to test concepts in a visual and intuitive manner through real time feedback. Simulation games have specifically attracted recent attention as learning experiences that straddle the pedagogical objectives and elements of fun to encourage student motivation and promote deeper learning. These simulation games or educational simulations are developed around a simplified model of reality or set of abstract concepts which students can visualize, test and reflect on (Dede and Lewis 1995; Galarneau 2004). What tends to make these learning experiences effective is that they allow students to fail, but through in-process reflection students can modify their strategies and repeat the process until reaching the goal. Hence, the competitive, interactive and fail-safe aspects of such learning experiences can increase their motivation and encourage students to invest time practicing their skills, deemed essential for effective learning (Aldrich 2003; Chen and Levinson 2006). Additionally, the application of simulation games in construction education has illustrated an increase in learners' motivation, problem-solving skills, and metacognitive processes (Castronovo et al. 2017b, 2018b; Lee et al. 2011).

Construction education research has been increasingly looking into virtual reality and game engines as platforms that bring interactive and motivational aspects to engage students in simulating various real-world scenarios for a range of tasks. Educational VR-based simulation in most instances is developed and used for design visualization, construction safety training, equipment operation and structural analysis (Wang et al. 2018). Used as an educational tool for exposing students and trainees to various safety scenarios (Le et al. 2015; Sacks et al. 2013), VRbased simulation consistently acts as a high-engagement form of training, which has been confirmed in prior meta-studies (Robson et al. 2010) to be more effective than low-engagement forms of training, such as lectures, images or videos. Specifically, in the study reported by Sacks et al. (2013), the semi-immersive power-wall setting offered significant advantages in terms of maintaining trainees' attention and concentration for stone cladding and cast-in-place concrete tasks, though less so for general safety knowledge. At the same time, the study raised questions around the suitability of group-based training using a power-wall for what should, in essence, rely on first-hand experience and thus, possibly the use of individual VR configurations. In their research Castronovo et al. (2017a) evaluated students' perceived value of performing VR design reviews and found that the VR systems had a significant impact on students' special presence, immersion, and enjoyment. These examples reveal how the pedagogical aspects require carefully crafted simulation scenarios where attention, motivation, and engagement are key to sustained learning. Equally, technological aspects of interactivity, feedback and immersion depend on the pedagogical goals and considerations that aim to place the learner in an active role, rather than as an observer.

\subsection{VIRTUAL REALITY IN CONSTRUCTION EDUCATION: THE DESIGN REVIEW SIMULATOR}

Virtual reality offers immense potential in education to recreate real-life scenarios and engage learners through its immersive, interactive and illustrative features. VR is distinguished from other more commonly used visualization mediums by the sense of closeness and control created by involvement: the feeling of presence that originates from an immersive, first-person 
view of digital information that responds to the users' movements and interactions with it (Nikolic 2007). Combining the pedagogical elements of simulation games that stimulate student learning, motivation, and problem-solving skills with the engaging experience of VR, the authors developed the VR educational game named Design Review Simulator (DRS) (Castronovo et al. 2019). The main learning objective of the game is for the students to learn to identify and evaluate the types of design-related mistakes that can be found in the BIM model of a San Francisco-based townhouse. During the gameplay, the students wear an immersive VR headset and are asked to walk around the townhouse and identify design mistakes pertaining to spatial layout, material use, coordination issues and missing components. Bloom's Taxonomy (Anderson and Krathwohl 2001; Bloom 1969) informed the development of the learning objectives with a specific focus on the cognitive domain and assessing the design review skills. Within this cognitive domain, 'remembering' and 'understanding' were selected as the primary cognitive thinking skills levels. Each of these levels was operationalized through action verbs, such as define, explain, match, and identify, aligned with a cognitive level of thinking skills set by the taxonomy. The four resulting learning objectives for the DRS thus focused on the ability for the students to identify, describe, match and explain the types of design mistakes following the completion of the game (see Table 1).

Table 1. Game Learning Objectives for the Design Review Simulator

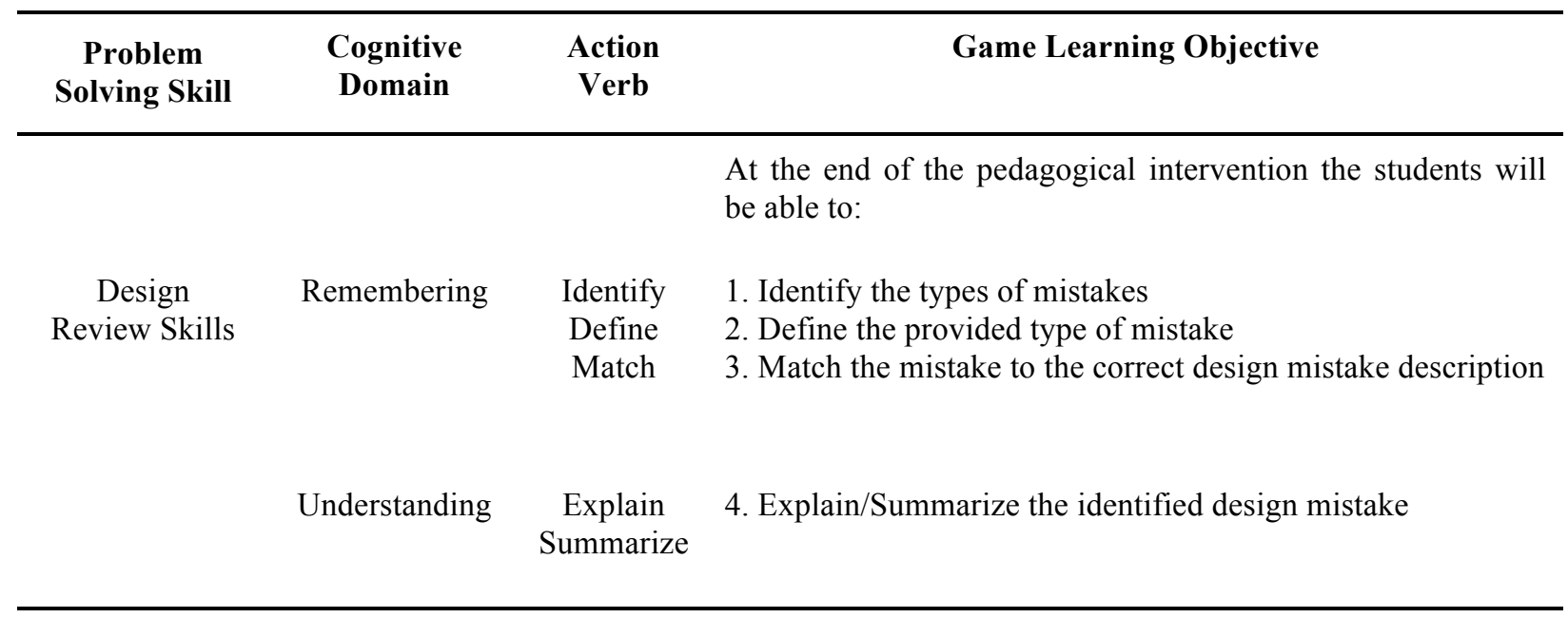

The game was designed to present the students with four types of design mistakes, for a total of 26 mistakes, each with a specific score associated with it. The point system was based on the severity of the type of mistake, including: (1) spatial layout, (2) coordination issues, (3) missing elements, and (4) improper material choices. Once in the game, the learners are first placed outside the townhouse and asked to look around and explore both inside and outside the building for any of the above types of issues (see Figure 1, left). To identify and flag a design mistake, the students point the crosshair to a design mistake and, using a keyboard, press "enter". If the mistake is correctly identified, the game displays a message with the type and description of the mistake (see Figure 1, right).

Initial evaluation of the DRS' impact in the classroom environment illustrated its potential in supporting students in gaining design review skills. In particular, through initial testing, the authors compared the students' performance in reviewing the design using the DRS 
and 2D drawings, learning that students using the DRS in a VR environment identified a significantly higher number of design mistakes than using traditional design documentation (Kandi et al. 2020). This warranted further investigation on the DRS' impact on knowledge and retention of skills through a larger sample size, in order to support the generalization of the findings.

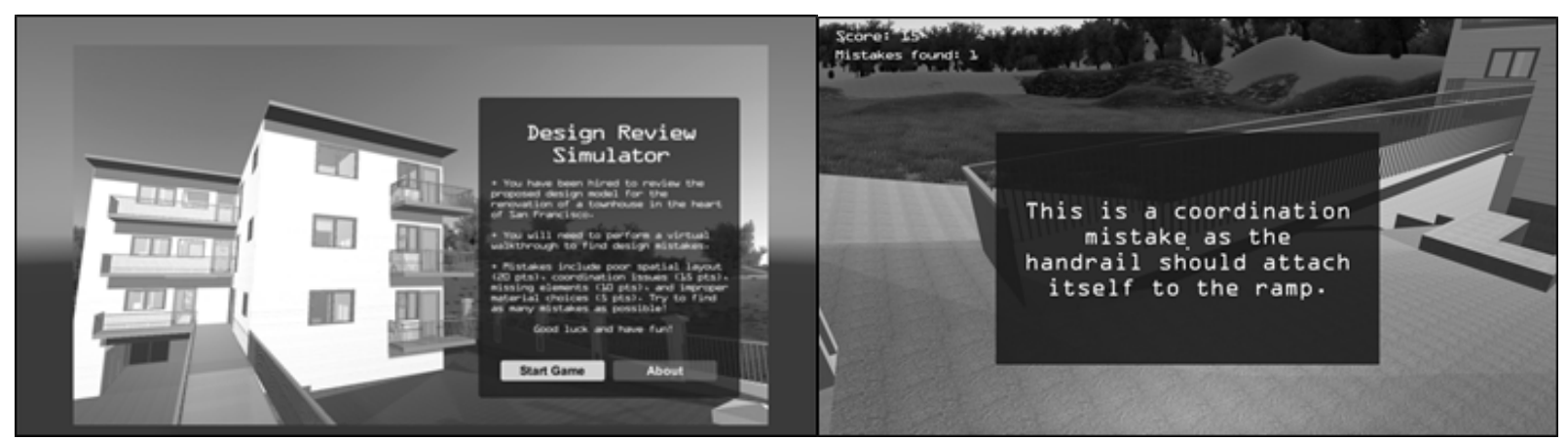

Figure 1. Design Review Simulator Game Play

\section{RESEARCH GOAL AND QUESTIONS}

The literature review highlighted current research and applications of engineering educational games using virtual reality. In particular, the Design Review Simulator VR educational game illustrated promise in having an impact on students' ability to identify design issues, though the small sample size limits the generalizability of the results and the claims of broader impact (Kandi et al. 2020). However, previous research in the implementation of the DRS requires the expansion of the sample size to generalize the findings. Additionally, the authors were interested in understanding which instructional implementation procedure would yield the highest learning impact. The authors were also interested in evaluating the impact of the game on the students' learning of design review skills.

To expand the sample size, the authors implemented the DRS in undergraduate courses in two North American institutions. To evaluate the impact of the DRS on students' skills in reviewing design, we sought to answer the following research questions:

1. What implementation procedure order yields the highest educational impact on students' ability to identify mistakes?

2. What implementation procedure order yields the highest educational impact on students' knowledge of the design review process?

3. Does playing the DRS simulation game lead construction students to identify a higher number of design mistakes than evaluating design on the drawings?

4. Does playing the DRS simulation game lead construction students to gain a higher knowledge of the design review process and related cognitive domains, such as remembering and understanding, than evaluating design on the drawings?

Based on these questions, the study tested the following null hypotheses, which are number-aligned to the research questions:

(1) The order with which the representations modes (i.e. VR and 2D drawings) are implemented will not have an impact on the students' ability to identify mistakes. 
(2) The order with which the representations modes (i.e. VR and 2D drawings) are implemented will not have an impact on the students' knowledge of the design review process.

(3) Students using the VR learning activity (DRS) will be able to recognize the same number of design mistakes as students using 2D drawings.

(4) Students using the VR learning activity (DRS) will gain the same knowledge of the design review process, such as remembering and understanding, as students using 2D drawings.

\section{METHODOLOGY}

\subsection{DESIGN AND PROCEDURE}

A mixed-design crossover repeated-measures experimental design was set up to evaluate and assess the design review skills that construction students gained from playing the DRS. This research design is known as a crossover experiment and it was chosen to control the potential sequencing effects of an independent variable and treatment contamination (Johnson 2010). This experimental design allows testing of both between-subject and within-subject differences (see Figure 2), as well as a comparison of the two groups to test the groups' equivalence.

Based on this design, recruited students were randomly assigned to two groups. The groups were then exposed to an independent variable (i.e. the learning medium) with two levels of treatment (i.e VR game versus 2D drawings). Each group completed both treatments on two consecutive days and at the same time, though the treatment sequence differed between the two groups. The first group used the 2D drawings on the first day and the VR game on the second day, while for the second group the sequence was reversed. The variation in sequence between the 2D drawings and VR game tests the possibility that student learning is not affected by such order.

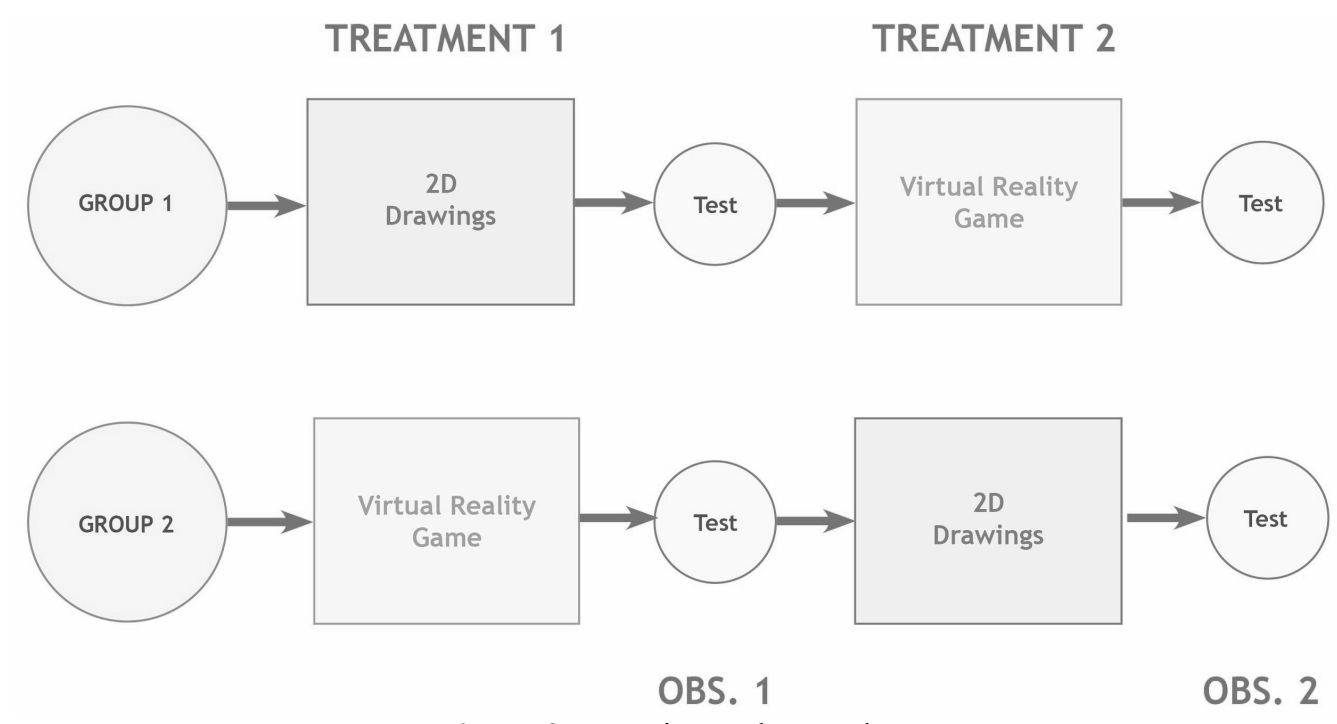

Figure 2. Experimental Procedure

During the experiment, the students were grouped in pairs (see Figure 3) and asked to review the design proposal of a sample building project through the provided learning medium; they were 
then asked to document the types of mistakes on a handout that was the same for both experimental groups. After each treatment, the student teams were asked to write down the mistakes on the handout. In particular, they had to take note of the number of mistakes they identified, the type of mistake as well as an explanation of why they chose that mistake type. The number of mistakes found by the students was used as the dependent variable of the experiment. Moreover, at the end of each treatment, the students were given an assessment instrument which included a set of ten multiple-choice or true-false types of questions (see Table 2 ), to test the students' achievement of the game's learning objectives (Table 1). The assessment of the design review-related knowledge gained by the students was used as another dependent variable. The dependent variables (i.e. knowledge assessment, number of mistakes) were then compared by performing two $2 \times 2$ mixed model ANOVA with the independent variable (i.e. learning medium) as the within-subjects factor (i.e. drawings, VR game) and with order as the repeated measure and condition as the between-subjects factor (groups: Group 1, Group 2).

Table 2. Assessment Instrument

\begin{tabular}{|c|c|c|}
\hline Learning Objective & Question Type & Question \\
\hline $\begin{array}{l}\text { 1. Identify the different types of } \\
\text { mistakes. } \\
\text { 2. Match the identified mistake to } \\
\text { the correct design mistake } \\
\text { description. }\end{array}$ & $\begin{array}{l}\text { Multiple } \\
\text { Choice }\end{array}$ & $\begin{array}{l}\text { 1. Which of the following is not a potential type of design } \\
\text { mistake? (Improper Material) } \\
\text { 2. The following is what type of mistake in the picture to } \\
\text { the right? (Coordination) } \\
\text { 3. The following is what type of mistake in the picture to } \\
\text { the right? (Spatial Layout) } \\
\text { 4. The following is what type of mistake in the picture to } \\
\text { the right? (Missing Element) }\end{array}$ \\
\hline
\end{tabular}

3. Define the identified design True or False mistake.

5. A material mistake can be defined as building elements that have the incorrect material assigned. $(\mathrm{T} / \mathrm{F})$

6. A coordination design mistake can be defined as building elements that are not positioned correctly in the design. (T/F)

7. A spatial layout design mistake can be defined as building elements that don't align with each other or interfere with each other. (T/F)

8. A design mistake can be defined as building elements that are not included or present in the design. (T/F)
4. Explain/Summarize the identified design mistake.
True or False
Multiple
Choice
9. Design review is an essential process to be performed during construction. $(\mathrm{T} / \mathrm{F})$
10. Evaluating design with $2 \mathrm{D}$ drawings is a process.

\subsection{PARTICIPANTS}

A total of 120 undergraduate students pursuing a construction management degree from two higher education institutions in Northern California participated in the study. The students 
were in their second or third year of studies. Institutional Review Board (IRB) consent was given to the authors to collect and use the data. The students were randomly split into two groups. The groups were assigned to have 60 students per group. However, 23 students had to be excluded from the analysis as they did not complete both treatments, either missing the first or second treatment. The final number of participants included 94 undergraduate construction management students.

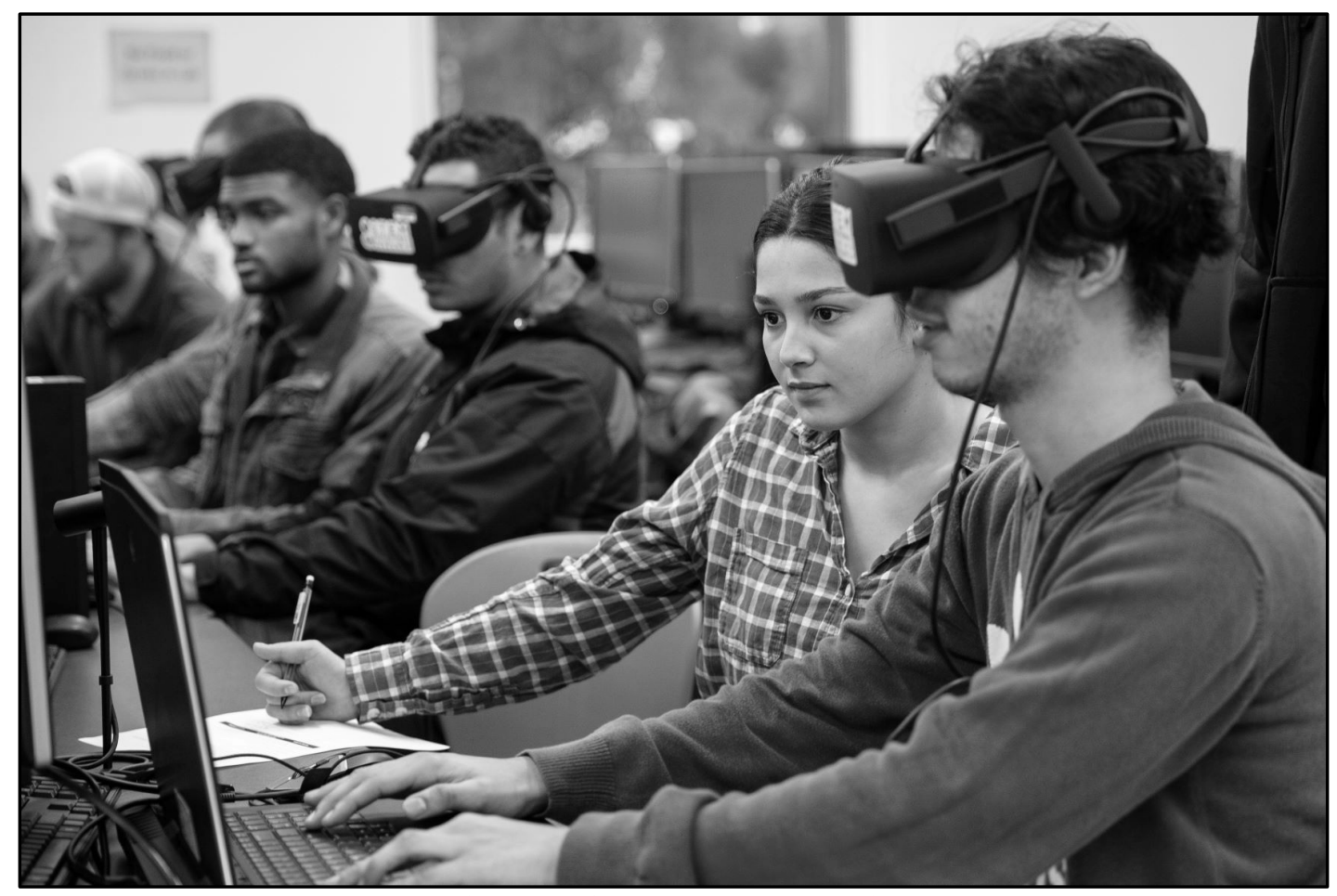

Figure 3. Students Working in Pairs During the VR Activity

\subsection{EQUIPMENT}

The authors used the DRS to assess the students' design review skills. To evaluate the gains in thinking skills and assess the students' design review skills, the authors used the data collected from the activity handout and the assessment instrument, composed of multiple-choice and true or false questions. The game was used in a classroom equipped with a variety of immersive and non-immersive VR tools including VR headsets, such as Oculus Rift and Lenovo Mirage, and VR-ready desktops. The student pairs using the 2D drawings were provided with the floor, elevation, and section plans of a sample building, and the paper handout to record their data. The student pairs using the VR game were provided with a VR headset to run the game and the paper handout to record their data. The sample building design proposal was the same in both treatments.

\section{RESULTS AND ANALYSIS}

The results from the experiment can be found in Table 3 and 4. The tables list the two levels of the independent variable, the learning medium to perform design reviews as a withinsubjects factor (2D drawings, VR game), the two dependent variables (knowledge assessment, 
number of mistakes), and order as the between-subjects factor (groups: Group 1, Group 2). For each of these variables, the means and standard deviation values are calculated. The data were analyzed using the statistical software package, IBM SPSS Statistics. In order to test the hypotheses, several analyses were conducted, such as two two-way mixed ANOVA followed by two independent T-Test and two paired-sample T-Tests. Two two-way mixed ANOVA were conducted to address the first and second hypothesis and whether the order of implementing the independent variable playing the DRS simulation game resulted in any difference in the dependent variables. In order to investigate further the group differences, two independent $\mathrm{T}$ Test was performed to evaluate the difference between groups' knowledge assessment scores. Two paired sample T-Tests were carried out to test the third and fourth hypothesis, that is if experiment resulted in higher knowledge or number of design mistakes identified.

Descriptive Statistics for Number of Mistakes

\begin{tabular}{|c|c|c|c|c|}
\hline & Groups & $\begin{array}{c}\text { Number Mistakes } \\
\text { Mean }\end{array}$ & Std. Deviation & $\mathbf{N}$ \\
\hline \multirow{3}{*}{$\begin{array}{c}\text { 2D Drawings-based } \\
\text { Assessment }\end{array}$} & Group1 & 4.6486 & 2.7306 & 37 \\
\hline & Group2 & 4.8947 & 1.5316 & 57 \\
\hline & Total & 4.7979 & 2.0769 & 94 \\
\hline \multirow{3}{*}{$\begin{array}{c}\text { VR-enabled } \\
\text { Assessment }\end{array}$} & Group1 & 11.1892 & 4.6057 & 37 \\
\hline & Group2 & 9.7368 & 3.3086 & 57 \\
\hline & Total & 10.3085 & 3.9131 & 94 \\
\hline
\end{tabular}

\begin{tabular}{|c|c|c|c|c|}
\hline & Groups & $\begin{array}{c}\text { Knowledge } \\
\text { Assessment Mean }\end{array}$ & Std. Deviation & $\mathbf{N}$ \\
\hline \multirow{3}{*}{$\begin{array}{c}\text { 2D Drawings-based } \\
\text { Assessment }\end{array}$} & Group1 & 44.3243 & 14.63 & 37 \\
\hline & Group2 & 58.4211 & 19.35 & 57 \\
\hline & Total & 52.8723 & 18.87 & 94 \\
\hline \multirow{3}{*}{$\begin{array}{l}\text { VR-enabled } \\
\text { Assessment }\end{array}$} & Group1 & 52.9730 & 15.61 & 37 \\
\hline & Group2 & 58.5965 & 17.87 & 57 \\
\hline & Total & 56.3830 & 17.16 & 94 \\
\hline
\end{tabular}

\subsection{IMPLEMENTATION ORDER ANALYSIS}

The authors aimed at testing hypothesis 1 and 2 which stated: "the order with which the representations modes (i.e. VR and $2 D$ drawings) are implemented will not have an impact on the students' ability to identify mistakes" and "the order with which the representations modes (i.e. VR and drawings) are implemented will not have an impact on the students' knowledge of the design review process". In order to test these hypotheses, two Two-Way Mixed ANOVA (2x2) analyses were conducted for each dependent variable. The Two-Way Mixed ANOVA (2x2) analysis was conducted using the independent variable as a within-subjects factor (paperbased 2D drawings and DRS VR game), and order, as a between-subjects factor (Group 1: (1) 
drawings, (2) VR game; and Group 2: (1) VR game, (2) drawings). The interpretation of these analyses is facilitated by graphing the four mean values. These are depicted in Figure 4, Mean of Number of Mistakes Results, and Figure 5 Mean Knowledge Assessment Scores Results.

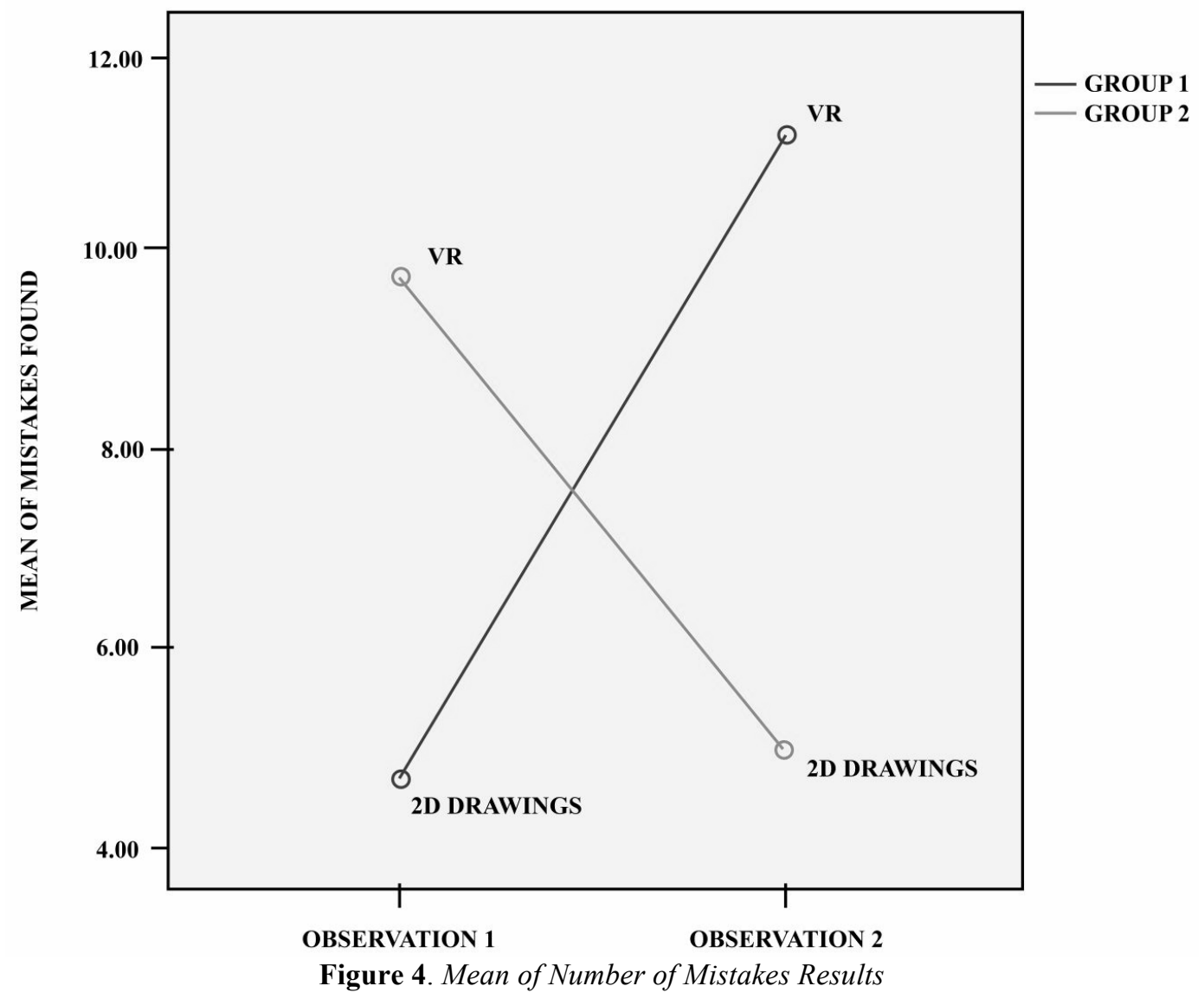

The results of the Two-Way Mixed ANOVA analysis for the number of mistakes found by the students showed that there was a significant difference in the within-subject factor $(F(1$, 92) $=4.776, p=0.049<0.05$, partial $\left.\eta^{2}=0.014\right)$, while there was not a significant difference in the between-subjects factor $\left(F(1,92)=1.302, p=0.257>0.05\right.$, partial $\left.\eta^{2}=0.014\right)$. On the other hand, the results of the Two-Way Mixed ANOVA analysis for the knowledge assessment showed that there was a significant difference in both the within-subject factor $(F(1,92)=4.79$, $p=.031<0.05$, partial $\left.\eta^{2}=0.5\right)$ and in the between-subjects factor $(F(1,92)=10.069, p=$ $0.002<0.05$, partial $\eta^{2}=0.099$ ). Moreover, the partial $\eta^{2}$ result for both ANOVA analysis indicates a middle effect of the sample size for the generalization of the results. In order to further analyze the within-subject factor results, further analysis was conducted. by performing paired sample T-Tests.

Based on these analyses, the authors failed to reject the null hypothesis that "the order with which the representations modes (i.e. VR and $2 D$ drawings) are implemented will not have an impact on the students' ability to identify mistakes" when looking at the number of mistakes identified by the students. This result illustrates that the order or between-subjects with which the independent variable, or medium, is implemented does not have an impact on the students' ability to identify mistakes. However, there is a difference of the within-subject factor, 
illustrating a difference in the number of mistakes identified when using one of the independent variables versus the other.

However, the authors can reject the null hypothesis that "the order with which the representations modes (i.e. VR and $2 D$ drawings) are implemented will not have an impact on the students' knowledge of the design review process" when looking at the students' knowledge assessment instrument scores. This result illustrates that the order with which the leaning medium is implemented does have an impact on the students' knowledge of the design review process (i.e. remembering and understanding cognitive domains). Additionally, there is a difference in the students' knowledge of the design review process when using one learning medium versus the other.

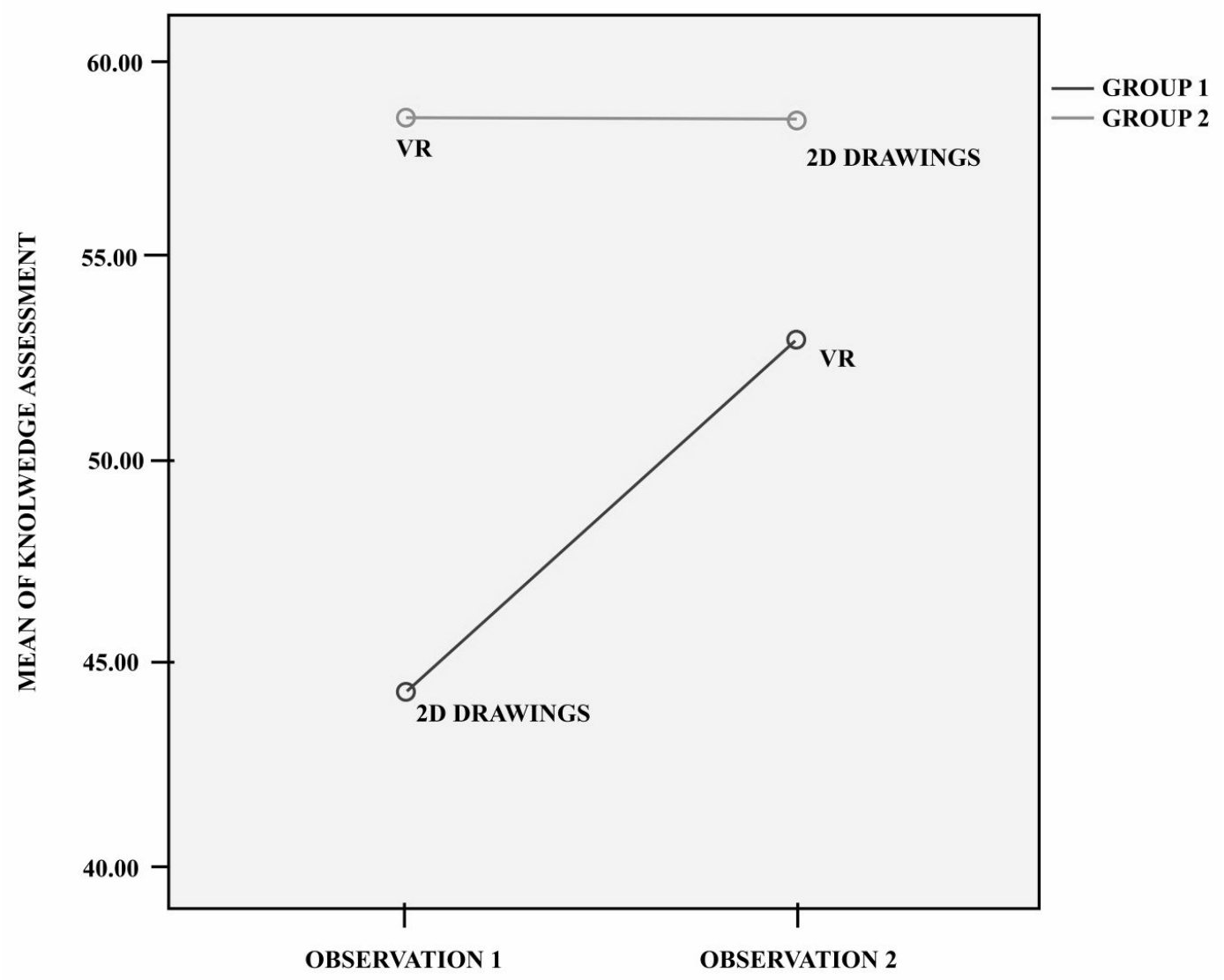

Figure 5. Mean Knowledge Assessment Scores Results

The authors further analyzed the group differences by performing an additional independent T-Test analysis. In this analysis, the authors wanted to test if there was any significant difference between students' final scores in the knowledge assessment after both treatment 1 and treatment 2 . The independent sample $\mathrm{T}$-Test showed that there was not a significant difference in the groups' final scores, $t(92)=1.435, p=0.155>0.05$ at $95 \%$ confidence interval, with a Cohen's $d=0.310$. Cohen's $d$ indicates a middle effect of the sample size for the generalization of the results. The lack of significant difference in the final results illustrates that at the end of the experiment, students achieved the same level of knowledge regardless of the order of implementation. 
The authors also wanted to investigate if there were any differences in the knowledge assessment scores for the students after they played the VR game, Group 1 VR scores versus Group 2 VR scores. The independent sample T-Test showed that there was not a significant difference in the groups' final scores, $t(92)=1.5649, p=0.121>0.05$ at $95 \%$ confidence interval, with a Cohen's $d=0.335$. Cohen's $d$ indicates a middle effect of the sample size for the generalization of the results. The lack of significant difference in the final results illustrates that after playing the VR game, the students achieved the same level of knowledge regardless of the order of implementation.

Lastly, the authors also wanted to analyze if there were any differences in the knowledge assessment scores for the students after they performed the 2D drawings reading activity, Group 1 Drawings scores versus Group 2 Drawings scores. The independent samples T-Test showed that there was a significant difference in the groups' final scores, $t$ (92) $=3.782, p=0.0003<$ 0.05 at $95 \%$ confidence interval, with a Cohen's $d=0.832$. Cohen's $d$ indicates a large effect of the sample size for the generalization of the results. The significant difference of the final results illustrates that the students who played the VR game first achieved a higher assessment score than the students who played the VR game second.

\subsection{MEETING LEARNING OBJECTIVES ANALYSIS}

In addition to evaluating the effect of the implementation order on student learning, the authors were interested in testing if the students met the learning objectives set for the activity in Table 1. Hypothesis 3, which stated that "students using the VR learning activity (DRS) will be able to recognize the same number of design mistakes as students using $2 D$ drawings", was tested first. To test this hypothesis a paired-sample T-Tests for the number of mistakes found by Group 1 was performed. For this analysis the assumption of normally distributed mean difference scores was examined and satisfied and the data were normally distributed. The results illustrated a significant difference in the students' average number of identified mistakes in Group 1 when going from 2D drawings to VR, $t(36)=7.3243, p=0.0001<0.05$ at $95 \%$ confidence interval, with a Cohen's $d=1.73$.

The authors compared the means of the students' knowledge results from Group 1. A paired-sample T-Test was performed to test hypothesis 4, which stated that: "students using the VR learning activity (DRS) will gain the same knowledge of the design review process, such as remembering and understanding, as students using $2 D$ drawings". For this analysis the assumption of normally distributed mean difference scores was examined and satisfied and the data was normally distributed. The analysis shows a significant growth in the students' average score on the knowledge assessment instrument (Table 2) when going from 2D drawings to VR, $t$ (36) $=2,4593, p=0.01<0.05$ at $95 \%$ confidence interval, with a Cohen's $d=0.56$. The sample size was found to be effective as shown by Cohen's $d$.

\section{DISCUSSION}

The presented study aimed at evaluating the impact of an educational virtual reality game for reviewing design in a classroom environment. In particular, the authors wanted to evaluate the impact of the DRS on the students' ability to identify design mistakes during the process of design review for a sample building. Furthermore, the goal of this study was to assess the DRS game's ability to support student learning and development of design review skills. Lastly, the 
authors wanted to evaluate the implementation procedure that would maximize the students' learning. To address the goals, the authors set forward a series of research questions and tested several hypotheses.

1) What implementation procedure order yields the highest educational impact on students' ability to identify mistakes? For the first question, no statistical difference in the group's means was found to support the null hypothesis that there is no difference in the order with which the treatments are implemented. Thus, regardless of whether the students were introduced to the DRS or 2D drawings first, the order did not yield a difference in their ability to identify mistakes. While this result could lead the authors to conclude that the order of implementation does not have an impact, the analysis of the students' knowledge brings a different outcome.

2) What implementation procedure order yields the highest educational impact on students' knowledge of the design review process? Unlike the results from the first research question, the analysis for the second question found there was a statistical difference in the group's means of their knowledge assessment. The analysis' result rejects the null hypothesis that the order did not have an impact on the students' knowledge of the design review process. Therefore, the analysis illustrates that the order with which the DRS is implemented has an impact on the students' knowledge and design review-related cognitive domains. A significant difference in the students' knowledge was found when they were introduced to the $2 \mathrm{D}$ drawings first, followed by the DRS. At the same time, the results illustrate that the students were able to transfer their skills onto the drawings when they played the DRS game first. The implication of the findings for the instructors for implementing the DRS game is that they could use it in the classroom before they use 2D drawings. In fact, playing the DRS first would not only allow students to achieve the highest knowledge assessment regarding the design review process, but it would support them in transferring such knowledge when evaluating 2D drawings.

3) Does playing the DRS simulation game lead construction students to identify a higher number of design mistakes than evaluating design on the drawings? The results revealed that the students found a significantly higher number of mistakes when playing the DRS game compared to 2D drawings. At the same time, after playing the DRS game students found a significantly lower number of mistakes when evaluating drawings. This is possibly due to the 2D drawings' inherent lack of a third dimension for evaluation and the additional effort the students needed to put forth when referencing elevations and sections drawings of the building. The implication of the findings is that the first person experience and full immersion acted as valuable spatial and perception cues for the students to be able to find a higher number of mistakes.

4) Does playing the DRS simulation game lead construction students to gain a higher knowledge of the design review process and related cognitive domains, such as remembering and understanding, than evaluating design on the drawings? For the fourth question, the data analysis shows that there is a significant difference in Group 1 students' knowledge scores when evaluating 2D drawings first. Group 1, who first performed 2D drawing evaluations, significantly improved their scores when they used the DRS. This illustrates that the DRS can support students in getting a higher score on knowledge assessments than when evaluating 2D drawings. The authors further investigated if the students' final knowledge scores were different between the groups. In particular, the authors tested if Group 1 final score after playing the DRS was different from Group 2 final score who performed the drawings' evaluation last. The final scores were not significantly different. This result supports the second research questions' 
finding on which order of implementation is best suited from the classroom. In particular, it illustrates that the students from Group 2 not only transferred their knowledge from the DRS to the drawings, but their score was also not significantly different from the students' Group 1 highest score.

\section{CONCLUSION}

The growing implementation of active and experiential learning methods in construction engineering education has led to the adoption of innovative technology in the classroom. One particular technology that is under scrutiny by several disciplines is VR. Meanwhile, VR has illustrated its value in performing design reviews in the industry (Castronovo et al. 2018a). However, VR benefits still have to be investigated in construction pedagogy. To address this research potential, the authors wanted to investigate the impact of an educational virtual reality game on learners' design review knowledge and skills. Based on the study presented, the authors were able to test the effects of an educational VR game, the Design Review Simulator, on students' achievement of a set of learning objectives such as to identify design mistakes and to match them to the correct mistake type. Additionally, the authors evaluated the optimal implementation procedure to achieve the highest educational impact also in terms of design review-related knowledge assessment.

The research results contribute to the growing knowledge base on the implementation of VR in the classroom. In particular, the research illustrated that the benefits of VR found in the construction industry in terms, for example, of improved communication, user involvement and feedback collection, could be translated into the classroom environment. Based on the results, the authors found that the DRS significantly supported the students in improving their skills in identifying mistakes and increased their knowledge of the design review process. Additionally, the analysis showed that the DRS could be implemented in an integrated manner with traditional representations; for example, if students use DRS before they are exposed to construction drawings, they will retain and transfer their knowledge when evaluating paper-based documentations such as 2D drawings. These findings are in line with other research in construction education (Castronovo 2016; Castronovo et al. 2017b; Nikolic et al. 2011; Nikolic and Windess 2019), which have found that construction educational games supported students in achieving learning objectives and transferring them into other media.

On the other hand, these findings do not advocate for the instruction of design review skills though VR solely. In fact, the use of 2D drawings before VR could fit with the theory of Productive Failure (Kapur 2008; Kapur and Bielaczyc 2012). Kapur and Bielaczyc (2012) believed that instructors must introduce students to complex problems to solve other problems, even if they were not able to succeed in solving them. Therefore, if an instructor wanted students to first use 2D drawings, they could be a primer or scaffold for the VR experience.

In addition to the scientific contributions, the authors also have developed a free to use open-source VR game and implementation educational material that future researchers and instructors can use in learning activities and for further research on that topic.

Furthermore, the current study presents a large sample size that supports the generalization of the results. However, additional research must be performed to further investigate the value of VR games in construction education. In future research, future medium differences could be investigated; for example, comparing other immersive and non-immersive virtual environments would support the authors in investigating the value of full-immersion in 
supporting design review learning. Additionally, the authors are interested in evaluating the impact of VR games in projects beyond residential housing to larger scope facilities, such as other building types or civil projects. Lastly, the authors would like to challenge learners in not only identifying design mistakes but also propose solutions to the problems found so as to evaluate the role of VR in problem-solving skills as well. Therefore, the authors want to go beyond Bloom's lower-order thinking skills such as remembering and understanding, which are within the scope of this paper, and investigate the game's impact on higher-order cognitive domains, such as analysis and evaluation.

\section{REFERENCES}

Aldrich, C. (2003). Simulations and the Future of Learning: An Innovative (and Perhaps Revolutionary) Approach to e-Learning. Jossey-Bass Inc., Publishers.

American Council for Construction Education (ACCE). (2014). Standards and criteria for accreditation for postsecondary construction education degree programs. Irving, TX.

Anderson, L. W., and Krathwohl, D. R. (2001). A taxonomy for learning, teaching, and assessing: a revision of Bloom's taxonomy of educational objectives. Longman, New York.

Bassanino, M., Fernando, T., and Wu, K.-C. (2014). "Can virtual workspaces enhance team communication and collaboration in design review meetings?" Architectural Engineering and Design Management, 10(3-4), 200-217.

van den Berg, M., Hartmann, T., and de Graaf, R. (2017). "Supporting design reviews with premeeting virtual reality environments." Journal of Information Technology in Construction (ITcon), 22(16), 305-321.

Bloom, B. S. (1969). Taxonomy of educational objectives: The classification of educational goals. Longman Group United Kingdom, United Kingdom.

Boton, C. (2018). "Supporting constructability analysis meetings with Immersive Virtual Reality-based collaborative BIM 4D simulation." Automation in Construction, Elsevier, 96, 1-15.

Bullinger, H.-J., Bauer, W., Wenzel, G., and Blach, R. (2010). "Towards user centered design (UCD) in architecture based on immersive virtual environments." Computers in Industry, Human-Centered Computing Systems in Industry - A Special Issue in Honor of Professor G. Salvendy, 61(4), 372-379.

Castronovo, Awad, B., and Akhavian, R. (2018a). "Implementation of Virtual Design Reviews in the Generation of As-Built Information." Construction Research Congress 2018, Proceedings, 285-294.

Castronovo, F. (2016). "Assessing Problem-Solving Skills in Construction Education with the Virtual Construction Simulator."

Castronovo, F., Nikolic, D., Liu, Y., and Messner, J. I. (2013). “An evaluation of immersive virtual reality systems for design reviews." London, U.K.

Castronovo, F., Nikolic, D., Mastrolembo Ventura, S., Shroff, V., Nguyen, A., Dinh, N., Yilmaz, S., Akhavian, R., and Gaedicke, C. (2019). "Design and Development of a Virtual Reality Educational Game for Architectural and Construction Reviews.” 2019 ASEE Annual Conference and Exposition Proceedings, Tampa, FL.

Castronovo, F., Oprean, D., Liu, Y., and Messner, J. (2017a). "Application of Immersive Virtual Reality Systems in an Interdisciplinary Design Studio Course." Lean and Computing in Construction Congress - Joint Conference on Computing in Construction. 
Castronovo, F., Van Meter, P. N., and Messner, J. I. (2018b). "Leveraging metacognitive prompts in construction educational games for higher educational gains." International Journal of Construction Management, 1-12.

Castronovo, F., Van Meter, P. N., Zappe, S. E., Leicht, R. M., and Messner, J. I. (2017b). "Developing Problem-Solving Skills in Construction Education with the Virtual Construction Simulator.” International Journal of Engineering Education, 33(2), 831846.

Cavazza, M., Charles, F., and Mead, S. J. (2004). "Developing re-usable interactive storytelling technologies." Building the Information Society, Springer, 39-44.

Chen, W., and Levinson, D. M. (2006). "Effectiveness of Learning Transportation Network Growth through Simulation." Journal of Professional Issues in Engineering Education and Practice, 132(1), 29-41.

Dede, C., and Lewis, M. (1995). Assessment of emerging educational technologies that might assist and enhance school-to-work transitions. Office of Technology Assessment, United States Congress, Washington, DC, 100.

Dunston, P. S., Arns, L. L., Mcglothlin, J. D., Lasker, G. C., and Kushner, A. G. (2011). “An immersive virtual reality mock-up for design review of hospital patient rooms." Collaborative design in virtual environments, Springer, 167-176.

Faas, D., Bao, Q., Frey, D. D., and Yang, M. C. (2014). "The influence of immersion and presence in early stage engineering designing and building." AI EDAM, Cambridge University Press, 28(2), 139-151.

Freina, L., and Ott, M. (2015). "A literature review on immersive virtual reality in education: state of the art and perspectives." The International Scientific Conference eLearning and Software for Education, 10-1007.

Galarneau, L. (2004). "The elearning edge: Leveraging interactive technologies in the design of engaging, effective learning experiences." Wellington, New Zealand.

Henry, D., and Furness, T. (1993). "Spatial perception in virtual environments: Evaluating an architectural application." Proceedings of IEEE Virtual Reality Annual International Symposium, IEEE, 33-40.

Jensen, L., and Konradsen, F. (2018). "A review of the use of virtual reality head-mounted displays in education and training." Education and Information Technologies, Springer, 23(4), 1515-1529.

Johnson, D. E. (2010). “Crossover experiments.” WIREs Computational Statistics, 2(5), 620625.

Kandi, V.R., Brittle, P., Gaedicke, C., Castronovo, F. (2020 Accepted). Application of a Virtual Reality Educational Game to Improve Design Review Skills. Construction Research Congress 2020 Proceedings.

Kapur, M. (2008). "Productive Failure.” Cognition and Instruction, 26(3), 379-424.

Kapur, M., and Bielaczyc, K. (2012). "Designing for Productive Failure.” Journal of the Learning Sciences, 21(1), 45-83.

Khashe, S., Becerik-Gerber, B., Lucas, G., and Gratch, J. (2018). "Persuasive effects of immersion in virtual environments for measuring pro-environmental behaviors." ISARC. Proceedings of the International Symposium on Automation and Robotics in Construction, IAARC Publications, 1-7.

Khemlani, L., Timerman, A., Bennen, B., and Kalay, Y. E. (1998). "Intelligent representation for computer-aided building design." Automation in Construction, Elsevier, 8(1), 49-71. 
Kirby, J. G., Cannalte, R. P., Hicks, D. K., and Japel, E. J. (1989). Constructibility and design reviews: analysis and recommendations for improvement. ARMY Construction Engineering Research Lab.

Lather, J. (2014). “Technology Enabled Spaces for High Performance Facilities.” 2nd Annual Building Energy Informatics Summit, Dec 8-9, 2014, Consortium for Building Engery Innovation, Philadelphia, PA.

Lather, J. I., and Messner, J. I. (2018). "Framework for a Hybrid Simulation Approach for an Integrated Decision Support System in Healthcare Facilities." 2018 Winter Simulation Conference (WSC), IEEE, Gothenburg, Sweden, 1406-1417.

Le Dantec, C. A., and Do, E. Y.-L. (2009). "The mechanisms of value transfer in design meetings." Design Studies, Elsevier, 30(2), 119-137.

Le, Q. T., Pedro, A., and Park, C. S. (2015). "A Social Virtual Reality Based Construction Safety Education System for Experiential Learning." Journal of Intelligent \& Robotic Systems, 79(3), 487-506.

Lee, S., Nikolic, D., Messner, J., and Anumba, C. (2011). "The development of the Virtual Construction Simulator 3: An interactive simulation environment for construction management education." Proceedings of the 2011 ASCE International Workshop on Computing in Civil Engineering, ASCE, Miami, FL, 454-461.

Leicht, R. M. (2009). "A framework for planning effective collaboration using interactive workspaces.” Doctoral Dissertation, The Pennsylvania State University, University Park, PA, USA.

Li, X., Yi, W., Chi, H.-L., Wang, X., and Chan, A. P. (2018). "A critical review of virtual and augmented reality (VR/AR) applications in construction safety." Automation in Construction, Elsevier, 86, 150-162.

Lin, J.-W., Duh, H. B.-L., Parker, D. E., Abi-Rached, H., and Furness, T. A. (2002). "Effects of field of view on presence, enjoyment, memory, and simulator sickness in a virtual environment." Virtual Reality, 2002. Proceedings. IEEE, IEEE, 164-171.

Litzinger, T. A., Lattuca, L. R., Hadgraft, R. G., and Newstetter, W. C. (2011). "Engineering education and the development of expertise." Journal of Engineering Education, 100(1), $123-150$.

Liu, Y. (2017). "Evaluating Design Review Meetings And The Use Of Virtual Reality For PostOccupancy Analysis.” Doctoral Dissertation, Pennsylvania State University.

Liu, Y., Lather, J., and Messner, J. (2014). "Virtual Reality to Support the Integrated Design Process: A Retrofit Case Study." Computing in Civil and Building Engineering (2014), American Society of Civil Engineers, 801-808.

Liu, Y., Messner, J. I., and Leicht, R. M. (2018). "A process model for usability and maintainability design reviews." Architectural Engineering and Design Management, 14(6), 457-469.

Liu Yifan, Castronovo Fadi, Messner John, and Leicht Robert. (2020). "Evaluating the Impact of Virtual Reality on Design Review Meetings." Journal of Computing in Civil Engineering, American Society of Civil Engineers, 34(1), 04019045.

Majumdar, T., Fischer, M. A., and Schwegler, B. R. (2006). "Conceptual design review with a virtual reality mock-up model." Building on IT: joint international conference on computing and decision making in civil and building engineering. Montreal, Canada: American Society of Civil Engineers, 2902-11.

Mastrolembo Ventura, S., Castronovo, F., and Ciribini, A. (2019). “A Framework of Procedural 
Considerations for Implementing Virtual Reality in Design Review."

Nikolic, D. (2007). "Evaluating Relative Impact Of Virtual Reality Components Detail And Realism On Spatial Comprehension And Presence." The Pennsylvania State University.

Nikolic, D., Jaruhar, S., and Messner, J. (2011). "Educational Simulation in Construction:

Virtual Construction Simulator." Journal of Computing in Civil Engineering, 25(6), 421429.

Nikolic, D., and Windess, B. (2019). "Evaluating immersive and non-immersive VR for spatial understanding in undergraduate construction education." Northumbria University, Newcastle, UK.

Okeil, A. (2010). "Hybrid design environments: immersive and non-immersive architectural design." Journal of Information Technology in Construction (ITcon), 15(16), 202-216.

Paes, D., Arantes, E., and Irizarry, J. (2017). "Immersive environment for improving the understanding of architectural 3D models: Comparing user spatial perception between immersive and traditional virtual reality systems." Automation in Construction, 84, 292 303.

Pinet, C. (1997). "Design evaluation based on virtual representation of spaces." CUMINCAD.

Robson, L., Stephenson, C., Schulte, P., Amick, B., Chan, S., Bielecky, A., Wang, A., Heidotting, T., Irvin, E., and Eggerth, D. (2010). "A systematic review of the effectiveness of training \& education for the protection of workers." Toronto: Institute for Work \& Health, 2010-127.

Sacks, R., Perlman, A., and Barak, R. (2013). "Construction safety training using immersive virtual reality." Construction Management and Economics, 31(9), 1005-1017.

Schaumann, D., Pilosof, N. P., Date, K., and Kalay, Y. E. (2016). "A study of human behavior simulation in architectural design for healthcare facilities." Annali dell'Istituto superiore di sanità, 52(1), 24-32.

Shiratuddin, M. F., and Thabet, W. (2007). "Information rich Virtual Environment (VE) design review." Maribor, Slovenia.

Wang, P., Wu, P., Wang, J., Chi, H.-L., and Wang, X. (2018). "A Critical Review of the Use of Virtual Reality in Construction Engineering Education and Training." International Journal of Environmental Research and Public Health, 15(6), 1204.

Whyte, J. (2003). "Innovation and users: virtual reality in the construction sector." Construction Management and Economics, 21(6), 565-572.

Whyte, J., and Nikolic, D. (2018). Virtual reality and the built environment. Routledge, Milton Park, Abingdon, Oxon; New York, NY.

Williams, K., and Pender, G. (2002). "Problem-Based Learning Approach to Construction Management Teaching." Journal of Professional Issues in Engineering Education and Practice, 128(1), 19-24. 